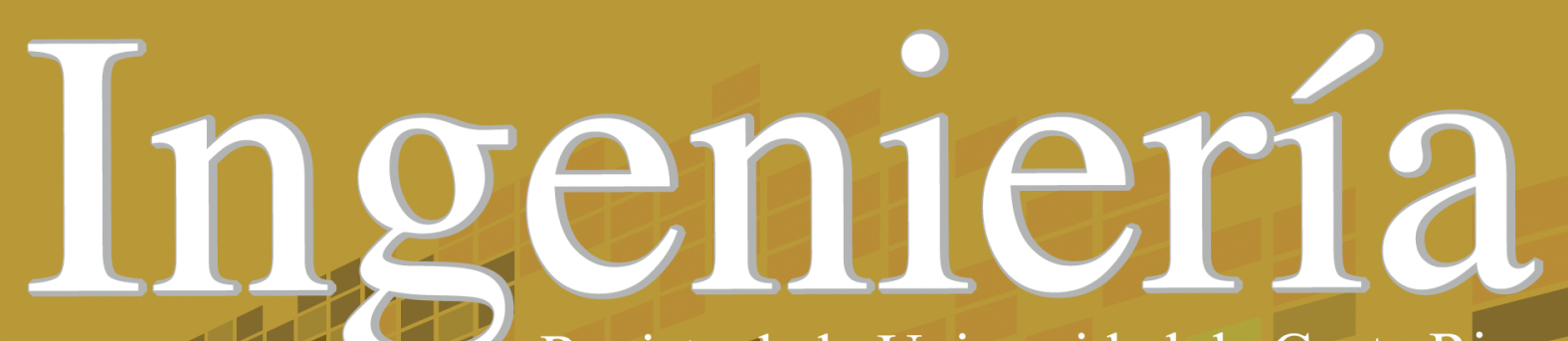

Revista de la Universidad de Costa Riea ENERO/JULIO 2013 - VOLUMEN 23 Número (1)

H

H

H71

Tr.

$x^{2}$

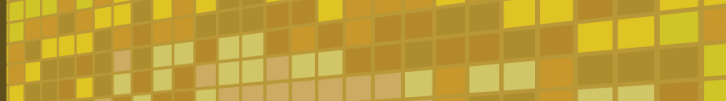

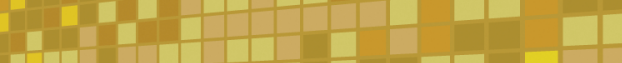

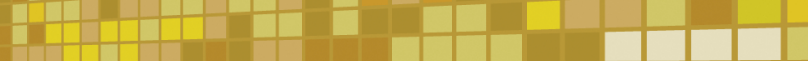

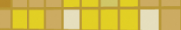

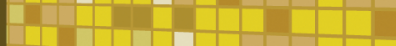

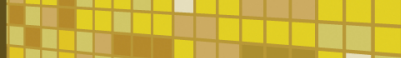

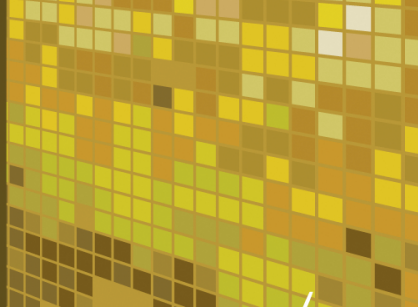

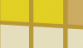



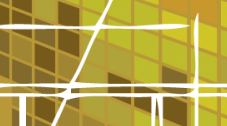

$+\infty \square$ 


\title{
MÉTODO PARA RECERRAR EL INTERRUPTOR DEL MEDIO EN SUBESTACIONES DE INTERRUPTOR Y MEDIO DEL SISTEMA ELÉCTRICO NACIONAL
}

\begin{abstract}
Resumen
Este artículo presenta una metodología para la función de auto-recierre en subestaciones de interruptor y medio del Sistema Eléctrico Nacional (SEN). Se propone controlar el interruptor del medio, o compartido, de manera tal que se ejecute un ciclo de auto-recierre sobre este interruptor únicamente cuando uno de los interruptores de línea se encuentra fuera de funcionamiento y una falla monofásica afecta la línea correspondiente. La metodología se basa en el monitoreo de las posiciones dobles de las cuchillas seccionadoras de los interruptores de línea y del medio.

Basado en el hecho que dicho algoritmo mejora la operación de subestaciones con esta configuración de barras, así como el hecho que dicho esquema de control se implementó en ciertas subestaciones del SEN (El Mogote, Nuevo Colón), el control del interruptor compartido es posible y una mejor maniobrabilidad podría ser obtenida. Por lo tanto, ante posibles obras de mantenimiento sobre un interruptor de línea, el interruptor compartido podría realizar un auto-recierre y preservar la integridad del sistema de potencia, cuando una falla monofásica en la línea de transmisión ocurre.
\end{abstract}

Palabras clave: Auto-recierre, diseño de control, interruptor de potencia, protección del sistema de potencia.

\begin{abstract}
This paper presents a methodology for the auto-reclose function in breaker-and-a-half substations of the Costa Rican electrical power system. It is proposed to control the middle breaker, in order to carry out an auto-reclose cycle on this circuit breaker only when one line breaker is in maintenance and when a single phase fault occurs on the corresponding transmission line. The method is based on monitoring the double position of the isolators on both the line and middle breaker.

Based on the fact that the algorithm improves the operation of the substations with this busbar arrangement and also that this algorithm is implemented in various substations in the Costa Rican electrical power system, the control of the middle breaker is possible and a better operability can be achieved. Therefore, under possible maintenance works on the line breaker, the middle breaker would be able to carry out the auto-reclose cycle and preserve the power systems integrity, when a single phase fault occurs on the transmission line.
\end{abstract}

Key words: Auto-reclose, circuit breaker, control design, power system protection.

Recibido: 16 de Agosto del 2012 • Aprobado: 11 junio del 2013

\section{INTRODUCCIÓN}

El Sistema Eléctrico Nacional (SEN) está constituido por cientos de subestaciones ubicadas en lugares específicos en Costa Rica, de modo tal y que cada zona del país tenga acceso al servicio eléctrico. La discusión de la correcta ubicación de dichas subestaciones no es el objetivo de este artículo de investigación. Tal y como se puede observar en el diagrama unifilar del SEN mostrado en la Figura 1, los niveles de tensión que el Instituto Costarricense de Electricidad utiliza para la transmisión de electricidad son: 138 kV y 230 kV (ICE-CENCE, 2012).

La Figura 1 muestra también muchas de las subestaciones de alta tensión del SEN. Desde 
el punto de vista de protección de la red, y considerando la criticidad de las subestaciones, éstas pueden ser clasificadas en tres grupos diferentes: no-críticas, semi-críticas o críticas. La designación del grado de criticidad de una subestación depende del impacto que se produce en el SEN cuando un elemento de dicha subestación se pierde. Dicha designación tiene un efecto importante en el diseño del esquema de protección en dicha subestación. Subestaciones con un alto nivel de criticidad requieren un alto nivel de confiabilidad en su operación, por lo tanto, los esquemas de protección en dichas subestaciones incluyen redundancia y diferentes funciones de respaldo.

Los esquemas comunes de barras, por ejemplo barra sencilla, barra partida, barra simple con reserva, han demostrado ser esquemas de barras muy robustos que han funcionado muy bien en el SEN. Sin embargo, y con el objetivo de aumentar los índices de confiabilidad del SEN, el Instituto Costarricense de Electricidad (ICE) ha iniciado recientemente la construcción de nuevas subestaciones con esquemas de barras denominados interruptor y medio (Breaker-AndA-Half) (ICE-CENCE, 2012). Una característica importante de los esquemas de barras de interruptor y medio es la continuidad en la operación de las líneas de transmisión, incluso, cuando hay un elemento de la subestación, interruptor o cuchilla seccionadora, fuera de servicio (D. Nack, 2005; L. Grigsby, 2006). Otra importante característica es que en caso de una perturbación sobre una de las bahías (de línea o de transformador), únicamente la bahía afectada es aislada y las restantes bahías mantienen su operación normal así como ambas barras. Cuando se habla de bahías de línea, se refiere a la presencia de una línea de transmisión que interconecta dicha subestación con aledañas. Por otro lado, cuando se habla de bahía de transformador, se refiere a la presencia de un transformador. Por ejemplo, la Figura 2 presenta tres bahías de línea (BL) y tres bahías de transformador (BT).

Debido a la proliferación de subestaciones con esquemas de barras de interruptor y medio, y teniendo en cuenta que futuras subestaciones en el SEN podrían ser construidas con este esquema de barras, es importante preparar soluciones a los problemas que serán introducidos por dichos esquemas de barras. Uno de los principales desafíos, introducido con dichos esquemas de barras, es el esquema de protecciones. Con la implementación de esquemas de interruptor y medio, esquemas de protecciones, como la protección por falla de interruptor, diferencial de barra, protección de distancia y la función de auto-recierre, han tenido que ser ligeramente modificadas (A. Phadke \& J. Thord, 2009; J. Cordero-Leiton, 2009). Esta última función, autorecierre, es la encargada de restablecer la línea de transmisión tiempo después de que ésta fue desconectada por la operación inmediata de una protección de línea (por ejemplo, la protección de distancia). Esta función se ejecuta en el SEN después de la operación monopolar debido a una falla monofásica. El ciclo de auto-recierre tarda generalmente $400 \mathrm{~ms}$, cuando no es bloqueada por alguna otra función de protección, para enviar la señal binaria de auto-recierre al interruptor que fue desconectado (ICE-APMG, 2011).

Respecto a la función de auto-recierre en esquemas de interruptor y medio, se ha notado que cuando el interruptor de línea se encuentra fuera de operación y una falla en la línea de transmisión ocurre, el interruptor del medio operará correctamente aislando la falla. Sin embargo, debido a la falta de control para autorecerrar este interruptor, la línea permanece abierta hasta que el operador de la subestación ejecute el comando de cierre manual. Esta acción, que también puede ser ejecutada desde el centro de control utilizando actuales protocolos de comunicación, puede tardar horas, poniendo en riesgo la estabilidad del sistema cuando los márgenes de estabilidad son bajos.

Para resolver el problema mencionado anteriormente en la función de auto-recierre en subestaciones con esquema de interruptor y medio, este artículo de investigación propone implementar un algoritmo de control sobre el interruptor del medio. De esta manera, un ciclo de auto-recierre puede ser ejecutado sobre el interruptor del medio únicamente cuando el interruptor de línea se encuentra en mantenimiento. La metodología se basa en el monitoreo de la doble posición de las cuchillas seccionadoras de los interruptores de línea y 


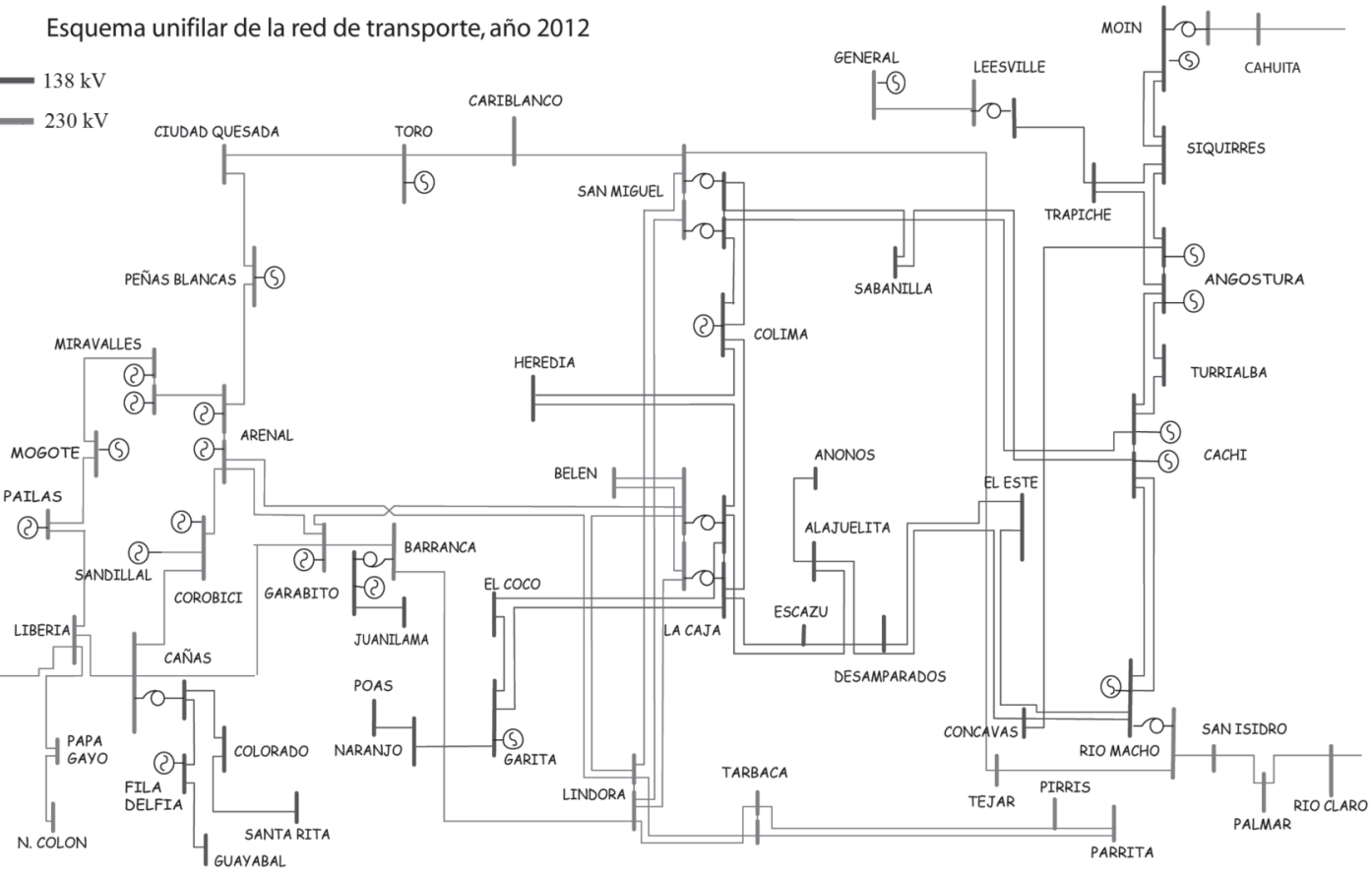

Figura 1. Diagrama unifilar del sistema eléctrico nacional.

Fuente: ICE-CENCE, 2012.

del medio. Luego, utilizando el protocolo de comunicación IEC 61850 para la automatización de subestaciones, y tomando en cuenta el uso de dispositivos electrónicos inteligentes, el algoritmo detecta el estado de ambos interruptores y utiliza el interruptor del medio cuando el de línea está fuera de operación.

El algoritmo ha sido efectivamente implementado en varias subestaciones del SEN (El Mogote, Nuevo Colon). En este artículo, el control de dicho interruptor se simula utilizando Matlab ${ }^{\circledR}$ y Simulink ${ }^{\circledR}$. Simulaciones se realizan para un segmento de una subestación de interruptor y medio en el SEN. Además de las variables de control, las simulaciones incluyen escenarios con perturbaciones sobre la línea de transmisión.

Este artículo está organizado de la siguiente manera: Sección 2: presenta una descripción de las subestaciones con esquema de interruptor y medio. Sección 3: define la función de autorecierre y detalles de otras funciones de protección en subestaciones de interruptor y medio. Sección 4: presenta la explicación y detalles de la metodología propuesta. Los resultados de las simulaciones son presentados luego en la Sección 5. Finalmente, Sección 6: presenta las conclusiones del artículo.

\section{SUBESTACIONES DE INTERRUPTOR Y MEDIO}

Las subestaciones con esquemas de barras de interruptor y medio se caracterizan por su alto nivel de confiabilidad. Estos esquemas han sido recientemente implementados en el SEN ya que su elevado costo representó un impedimento anteriormente. El Cuadro 1 muestra el nombre de las subestaciones en el SEN que han sido construidas con dicha configuración de barras.

La Figura 2 presenta el diagrama unifilar de un esquema de barras de interruptor y medio. Como se puede notar, este esquema cuenta con tres interruptores en cada diámetro. Un diámetro es la sección del esquema que comprende los elementos desde la "Barra A" hasta la "Barra 
B". Durante la operación normal, todos los interruptores permanecen cerrados y por lo tanto las dos barras se encuentran activas o energizadas. Este factor hace que el esquema de barras de interruptor y medio tenga mayor maniobrabilidad sobre otros esquemas.

Analizando individualmente cada bahía (de línea o de transformador) cuenta con dos interruptores. Sin embargo, uno de estos es compartido con la bahía adyacente. Al aumentar el número de elementos y al cambiar el esquema tradicional utilizado en el SEN, los criterios para los esquemas de protección, selección de la tensión de referencia para sincronización y la lógica de enclavamientos, cambian significativamente respecto a los diseños anteriores (J. Cordero-Leiton, 2009). Por lo tanto y como se puede inferir, la implementación de estos nuevos esquemas de barras ha conllevado a la obtención de nuevos desafíos, que a su vez se refleja en el desarrollo de nuevas soluciones.

Un mayor detalle de un diseño recomendado por el ICE, incluyendo la colocación de los transformadores de corriente y tensión de un diámetro, se presenta en la Figura 3. Esta figura será también utilizada posteriormente como referencia para el análisis de la lógica propuesta.

\section{FUNCIÓN DE AUTO-RECIERRE EN EL SEN Y PROTOCOLO IEC 61850}

\subsection{Función de auto-recierre}

La función de auto-recierre de interruptor (ANSI 79) permite realizar maniobras que restablezcan el sistema de potencia, por lo que es una consideración importante a realizar. $\mathrm{Al}$ realizar un auto-recierre, se busca restablecer el sistema de la manera más pronta posible, logrando así la continuidad del mismo. Un ciclo de auto-recierre puede llevarse a cabo en el SEN únicamente cuando los interruptores son monopolares. En caso contrario, dicha función deberá ser deshabilitada. Al momento de escribirse este artículo, el ICE analiza la posibilidad de implementar la función de autorecierre incluso cuando los interruptores son tripolares. La función de auto-recierre deberá

Cuadro 1. Subestaciones en el SEN con esquema de barras de interruptor y medio.

\begin{tabular}{cccc}
\hline $\begin{array}{c}\text { Nombre de la } \\
\text { subestación }\end{array}$ & $\begin{array}{c}\text { Nivel de tensión } \\
(\mathbf{k V})\end{array}$ & $\begin{array}{c}\text { Ubicación en el } \\
\text { SEN }\end{array}$ & $\begin{array}{c}\text { Año de } \\
\text { construcción }\end{array}$ \\
\hline Garabito & 230 & Zona Norte & 2010 \\
Mogote & 230 & Zona Norte & 2010 \\
Nuevo Colón & 230 & Zona Norte & 2010 \\
Pailas & 230 & Zona Norte & 2011 \\
Papagayo & 230 & Zona Norte & 2010 \\
Parrita & 230 & Zona Sur & 2009 \\
Pirrís & 230 & Zona Sur & 2011 \\
Venecia & 230 & Zona Central & 2012 \\
\hline
\end{tabular}




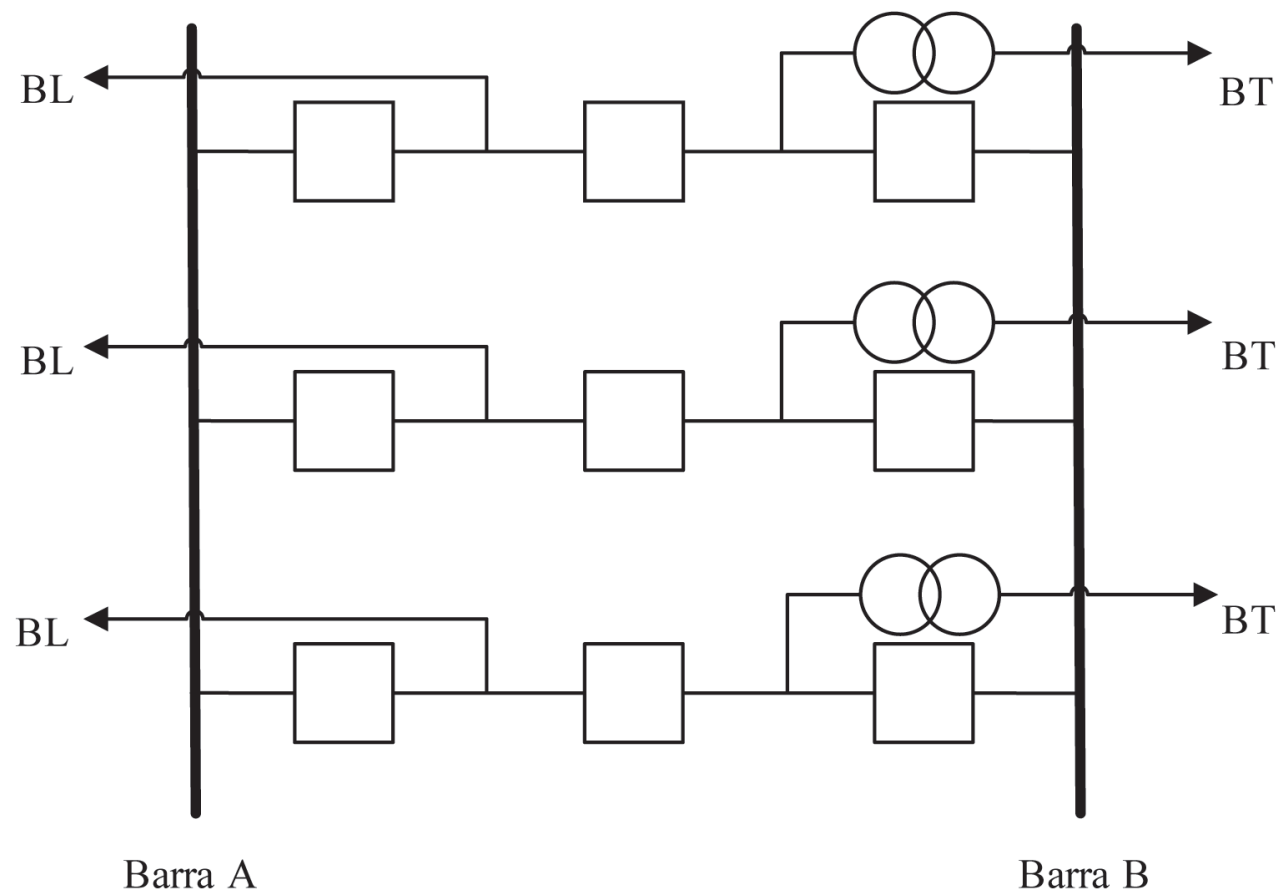

Figura 2. Diagrama unifilar de un esquema de barras de interruptor y medio con tres diámetros.

Fuente: Elaboración propia, 2012.

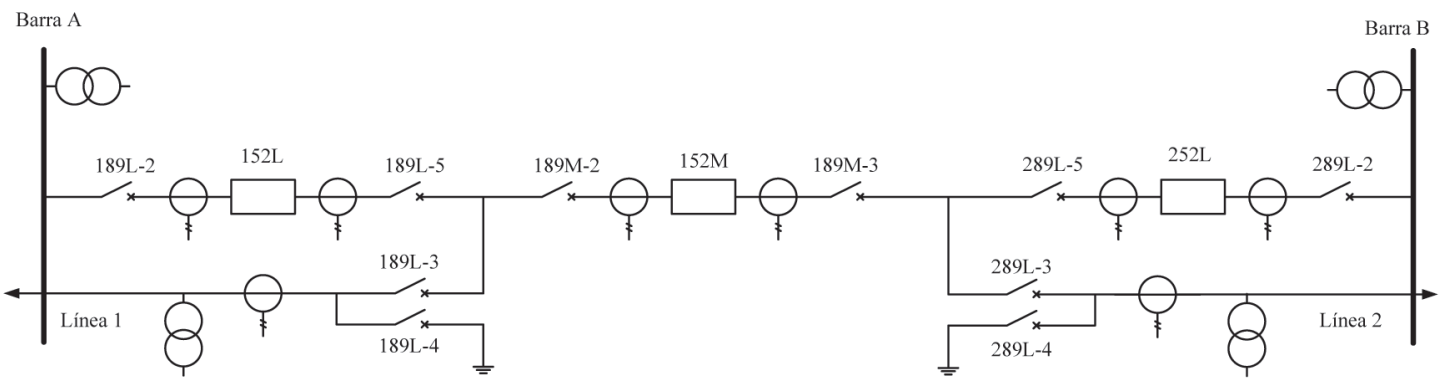

Figura 3. Representación detallada del diámetro de un esquema de barras de interruptor y medio.

Fuente: Elaboración propia, 2012.

ser deshabilitada cuando se cuenta con una bahía de transformador. Lo anterior se debe a las altas corrientes de magnetización.

En adelante, se considera el escenario en el cual una perturbación ocurre en la "Línea 1". El análisis llevado a cabo puede también realizarse para cuando la perturbación afecta la "Línea 2". Basados en la Figura 3, en el momento en que una falla monofásica ocurre, la protección de distancia o la diferencial de línea enviarán un comando de disparo al interruptor de línea 152L y al interruptor compartido 152M. Debe quedar claro que se analiza únicamente el caso en el que "Línea 1" es efectivamente una bahía de línea. En el caso que no se recibe ninguna señal de bloqueo para la función de auto-recierre, esta función se ejecutará 400 $\mathrm{ms}$ posterior al disparo, esto asumiendo que una operación monopolar fue producida. Analizando el 
caso en el que el interruptor de la "Barra A" (152L) y el interruptor compartido (152M) se encuentran abiertos posterior al disparo, la función de autorecierre ejecutará el recierre al interruptor de línea 152L. Dejando el interruptor compartido 152M abierto, hasta que el operador de la subestación, o vía comando remoto desde el centro de control, ejecute el comando de cierre manual.

Bajo esta circunstancia, se podría pensar que no hay ningún problema. Sin embargo, una importante dificultad se encuentra cuando el interruptor de línea $152 \mathrm{~L}$ se encuentra en mantenimiento. En este caso, si ocurre una falla en la misma línea el interruptor compartido no sería auto-recerrado, ya que no se contaba, en ese momento, con un algoritmo que permita el auto-recierre de dicho elemento. Para resolver este problema, la Sección 4 presenta el algoritmo propuesto para controlar el interruptor del medio, de manera tal que ejecute un ciclo de auto-recierre sobre el interruptor compartido únicamente cuando el interruptor de línea se encuentra en mantenimiento.

La función de auto-recierre se utiliza para cerrar una línea tan pronto como sea posible cuando la línea fue disparada debido a una falla monofásica. En otras palabras, esta función se utiliza para restaurar el sistema a su configuración normal de funcionamiento. Específicamente, la función de auto-recierre es una característica de la protección para restaurar la línea después de cualquier perturbación momentánea, por ejemplo un rayo.

A. Phadke \& S. Horowitz (2008) describen cuatro diferentes tipos de regímenes. El primer esquema presenta un enfoque de un único autorecierre de alta velocidad. Este representa un auto-recierre automático sin tiempo de retardo. El segundo toma en cuenta un tiempo muerto después de recibida la señal de disparo. Esta se lleva a cabo para disipar el aire ionizado y los arcos de falla. La tercera considera un auto- recierre sincronizado. En otras palabras, un recierre automático con relés de verificación de sincronización para confirmar que las diferencias entre variables eléctricas de las dos subestaciones del sistema de transmisión está dentro de los valores predefinidos. El último tipo de régimen se refiere a un esquema de enclavamiento. Este bloqueo se utiliza cuando un cierto período las condiciones del sistema o elementos están presentes antes de volver a cerrar.

\subsection{Protocolo de comunicación IEC 61850}

El protocolo de comunicación IEC 61850 es un nuevo estándar internacional para la automatización de subestaciones (IEC, 200405; R. Mackiewicz, 2006). Este estándar explica cómo los protocolos de comunicación en las subestaciones están diseñados y construidos. La norma IEC 61850 es una parte de la International Electrotechnical Commission (IEC) el Comité Técnico 57 (TC57), la arquitectura de sistemas de energía eléctrica.

El enfoque considerado por las normas de TC57, incluyendo IEC 61850 es innovador y requiere una nueva forma de pensar acerca de la automatización de subestaciones. Esto se ha traducido en mejoras muy significativas en los costos y el rendimiento de las redes eléctricas. Esta norma se divide en 10 secciones (IEC, 2004-05). Parte 1 y 2 introducen la norma con una visión general y un glosario de términos. Los capítulos del 3 al 5 identifican los requisitos generales y específicos de la comunicación en una subestación desde un punto de vista funcional. Estos requisitos se utilizan para identificar los servicios y modelos de datos necesarios. La Sección 7 trae la definición de los servicios abstractos y la abstracción de los objetos de datos. En las siguientes secciones, definiciones sobre la asignación del objeto abstracto de datos y servicios en la especificación de mensajería de fabricación y en los valores de la muestra de medición. Finalmente se define una metodología para determinar la conformidad.

\section{METODOLOGÍA PROPUESTA PARA LA FUNCIÓN DE AUTO-RECIERRE}

En esta sección, se plantea y desarrolla la metodología planteada para la función de auto-recierre en subestaciones con esquema de interruptor y medio. La metodología se basa en hacer que el interruptor del medio (152M de la Figura 3) sea utilizado como interruptor de línea, cuando el interruptor de línea (152L o 252L) se encuentra en mantenimiento o que por algún otro motivo se encuentra fuera de operación.

Hoy en día, el actual funcionamiento de la función de auto-recierre en las subestaciones 
de interruptor y medio consiste en re-cierre del interruptor de línea (152L o 252L en la Figura 3). Lo anterior usualmente ocurre unos segundos e incluso milisegundos después de la perturbación. A lo anterior se le suma que el interruptor del centro (152M en la Figura 3) queda abierto hasta que la señal de cierre manual sea recibida. Este tipo de funcionamiento ocurre en la mayoría de las subestaciones del SEN. En la práctica, los relés (por ejemplo reles de impedancia) poseen la opción de ejecutar recierres. Sin embargo, esta señal se envía, en el SEN, únicamente al interruptor de línea. La necesidad de ejecutar recierres en el interruptor del medio fue identificada y resuelta por el autor durante investigación conjunta con el personal del ICE, Guanacaste.

Enfocados en la "Línea 1", la metodología desarrollada propone resolver el problema en el caso cuando el interruptor de 152L está en mantenimiento. Para explicar la metodología, se considera todos los elementos cerrados (en todas las fases). También, se considera comunicaciones por fibra óptica en la subestación. La metodología no debería llevarse a cabo utilizando otro tipo de comunicación. Lo anterior se debe a que otros tipos de comunicación introducirían retrasos inaceptables cuando se trata de proteger el sistema de potencia. Por lo tanto, se identificó que el método seria vulnerable a la falta de canales de comunicación.

\subsection{Descripción de la lógica}

Como se puede observar en la Figura 4, la lógica propuesta es simple y consiste en puertas lógicas (AND, OR, inversores y flipflops). La señal de disparo se asume fue enviada desde el dispositivo de protección (por ejemplo protección de impedancia) para abrir los dos interruptores: 152L y 152M. Además, la señal de auto-recierre automático es enviada por la función de auto-recierre (ANSI 79).

Como se puede apreciar, el monitoreo de la doble posición de las cuchillas seccionadoras, 189L-2 y 189L-5 para el interruptor 152L y, $189 \mathrm{M}-2$ y $189 \mathrm{M}-3$ para el interruptor $152 \mathrm{M}$, es relevante para el buen funcionar de la metodología propuesta. En sistemas de potencia reales, estas cuchillas seccionadoras pueden ser controladas y monitoreadas mediante el uso de protocolos de comunicación basados en el Estándar IEC 61850 y el correspondiente uso de los adecuados canales de comunicación. Es importante mencionar que las indicaciones de posición de las cuchillas seccionadoras, así como de los interruptores, son dobles. En otras palabras, estas mediciones indican la posición de abierto o la posición de cerrado del elemento correspondiente.

En general, todas las cuchillas seccionadoras e interruptores se asumen estar cerrados en las tres fases. Así, la posición de cerrado se comprueba a través de una compuerta lógica AND. Es evidente en la Figura 4 que la señal de reenganche automático se envía a los dos interruptores $152 \mathrm{~L}$ y $152 \mathrm{M}$, sin embargo, y utilizando la combinación adecuada de compuertas y la correspondiente supervisión de la posición de las cuchillas seccionadoras, es posible recerrar el debido interruptor. Cuando todas las cuchillas seccionadoras están cerradas, la señal de recierre automático es recibida únicamente por el 152L, y cuando las cuchillas seccionadoras 189L-2 y/o 189L-5 están abiertas, esta señal es recibida por el interruptor $152 \mathrm{M}$.

En primer lugar, es necesario hacer notar que las simulaciones se harán considerando una falla monofásica en la "Línea 1". Cuando la falla ocurre, la señal de disparo es enviada a los interruptores $152 \mathrm{~L}$ y $152 \mathrm{M}$ para aislar la falla. $\mathrm{La}$ señal de disparo es enviada únicamente al polo correspondiente a la falla. Ejemplo, si la falla es monofásica en la fase A, el polo correspondiente a esta fase será desconectado. Asumiendo que la operación ideal del sistema, el ciclo de recierre automático inicia y milisegundos o segundos más tarde (en nuestro caso $400 \mathrm{~ms}$ ) el interruptor 152L es cerrado debido a la señal analógica enviada por el dispositivo de recierre automático. Esto sucederá únicamente si la función de recierre no recibe una señal de bloqueo. El interruptor 152M disparará aproximadamente a los 3 segundos debido a que no fue recerrado automáticamente y la protección por discrepancia de fases envía un disparo a los polos que permanecían conectados en el interruptor 152M (en nuestro ejemplo, las fases $B$ y $C$ ).

Durante la operación normal de la subestación es evidente que el interruptor 152M no debe recerrar automáticamente después de una perturbación. Este interruptor será manualmente cerrado mediante el envió de una señal análoga 
desde el centro de control. En este sentido, la dificultad comienza cuando el interruptor $152 \mathrm{~L}$ está en mantenimiento.

De acuerdo con el texto anterior y la necesidad de restaurar el sistema tan pronto como sea posible, se propone una nueva y práctica metodología para recerrar la "Línea 1" mediante un recierre automático del interruptor $152 \mathrm{M}$ cuando el interruptor 152L está fuera de operación.

De acuerdo con el método propuesto, que es factible para la integridad del sistema, para recerrar automáticamente el interruptor $152 \mathrm{M}$, se requieren tres señales diferentes: (i) la señal de recierre automático, (ii) las señales de indicación cerrado de las cuchillas seccionadoras 189M-2 y 189M-3, y (iii) las señales de indicación abierta de las cuchillas seccionadoras 189L-2 y/o189L-5.

De acuerdo a los códigos de seguridad, se considera que cuando un interruptor será puesto en mantenimiento, el personal de mantenimiento abrirá las cuchillas seccionadoras adyacentes a dicho interruptor (en este caso, si operan el interruptor 152L, las cuchillas seccionadoras 189L-2 y 189L-5 deberán ser abiertas). Así, ambos contactos perderán la indicación de "189L-2 CERRADO" y "189L-5 CERRADO", y la indicación cambiará para indicar interruptores abiertos. Por el contrario, el interruptor 152M seguirá operando y las cuchillas seccionadoras 189M-2 y 189M-3 estarán cerradas. Con estas combinaciones, el interruptor $152 \mathrm{M}$ funcionará como interruptor de línea.

Es importante mencionar que la señal de reset puede ser obtenida en aplicaciones reales, considerando la señal de indicación de que el ciclo de recierre ha finalizado. Entonces, una vez que el ciclo de recierre finaliza, la señal de reset se envía a los flip-flops y estos quedarían listos para una futura operación.

\section{RESULTADO DE SIMULACIONES}

Para demostrar la validez de la lógica propuesta serán mostradas las indicaciones de falla, de auto-recierre y las posiciones binarias de los interruptores. Además, serán mostrados el valor de la corriente por las líneas de transmisión y las tensiones eléctricas durante las simulaciones. Se asume que una perturbación ocurre a $450 \mathrm{~ms}$ y que la señal de auto-recierre, en caso de ser enviada, se envía 400 ms después del disparo. Estas simulaciones se han llevado a cabo en Matlab® y Simulink ${ }^{\circledR}$ considerando el valor de "0" como inactivado o abierto y "1" como activado o cerrado.

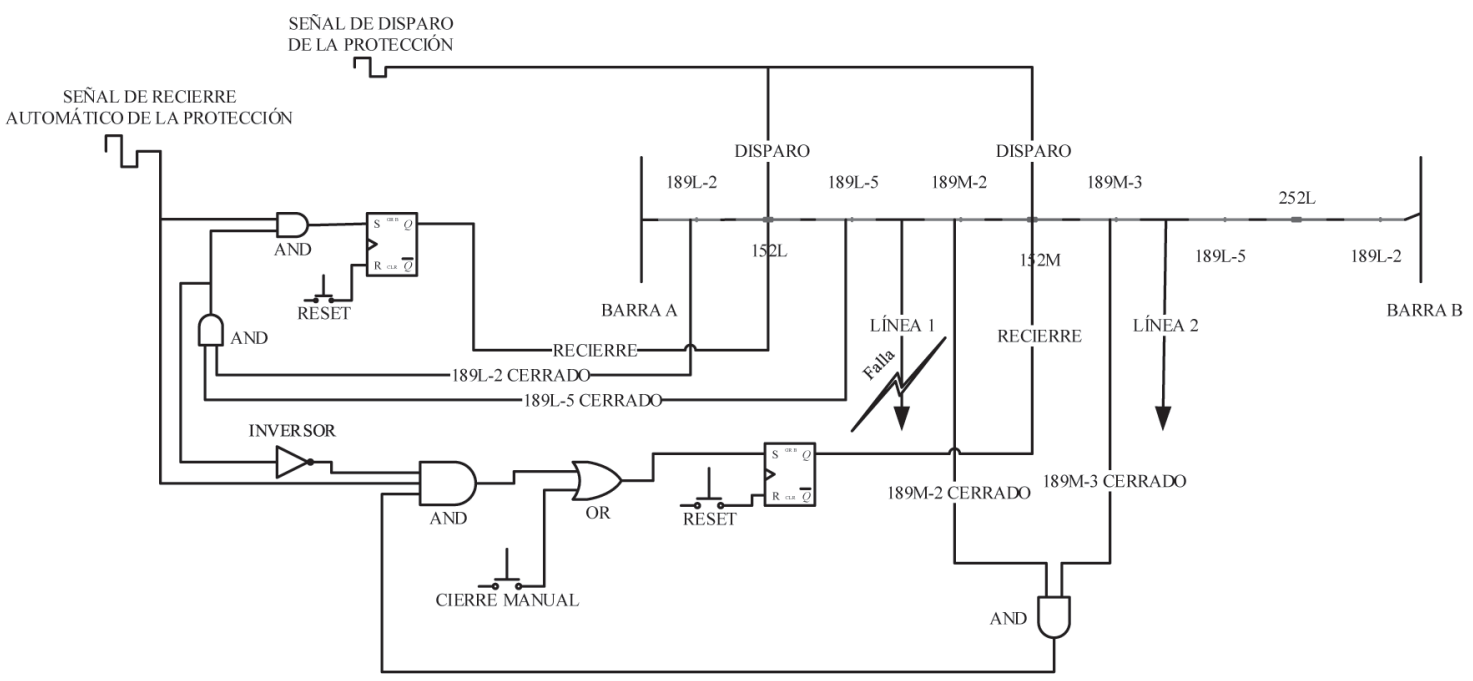

Figura 4. Metodología propuesta para recerrar el interruptor $152 \mathrm{M}$, cuando uno de los interruptores de línea (152L o 252L) está en mantenimiento.

Fuente: Elaboración propia, 2012. 
En sistemas de potencia reales, el método funcionará únicamente cuando una falla en el sistema ocurre. Cuando la falla ocurre, la señal de disparo es enviada por un dispositivo de protección para despejar la falla. La señal de disparo es normalmente enviada por la protección de impedancia (ANSI 21). Sin embargo, este artículo de investigación validará el método obviando los niveles de corriente. En su lugar, se presentarán corrientes para representar que los transformadores de corriente están efectivamente midiendo un valor de corriente a través del elemento. Debido a que el objetivo del artículo es presentar la aplicación de un nuevo método para mejorar la operación de la función de auto-recierre en las subestaciones de interruptor y medio del SEN, se considera que las cargas conectadas a través de las "Línea 1" y "Línea 2" son muy similares, por lo tanto, la corriente a través del interruptor 152M es muy baja. Esto es únicamente para validación del método. En sistemas de potencia reales, la falla ocurrirá y las corrientes variaran de acuerdo a los niveles de cortocircuito y la distancia de la falla a las fuentes. Entre más largo esté la falla de la fuente, más bajo serán las corrientes de cortocircuito. Además, las corrientes a través de las líneas de transmisión son difícilmente

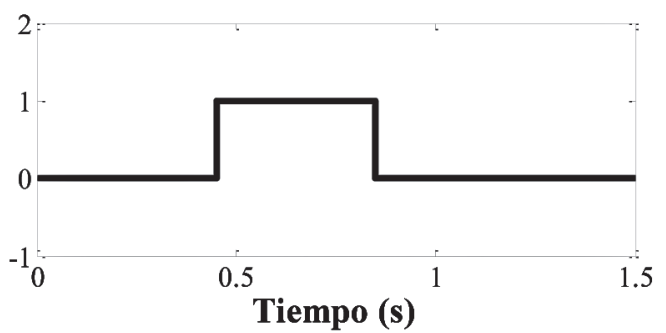

(a)

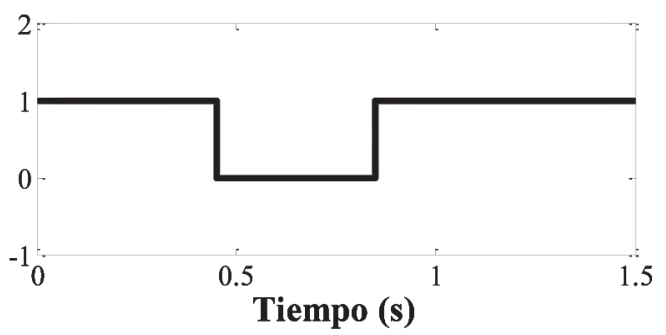

(c) similares. Aunque las subestaciones de interruptor y medio no están equipadas con un transformador de tensión para medir la tensión eléctrica directamente en el interruptor $152 \mathrm{M}$, se asumirá que existe dicho interruptor y que dicha tensión puede ser medida. Esta medición se denotará como tensión eléctrica en el interruptor $152 \mathrm{M}$.

\subsection{Simulaciones con el Interruptor de línea 152L operando normalmente}

Las Figuras 5 y 6 representan el funcionamiento normal del sistema, es decir, cuando el interruptor 152L está funcionando. En general, este interruptor está cerrado y la señal de disparo abre ambos interruptores $152 \mathrm{~L}$ y $152 \mathrm{M}$. Sin embargo, la señal de auto-recierre es recibida por el interruptor de línea y la corriente fluye solamente a través del interruptor 152L. Para lo que sigue de la Sección 5.1, las cuchillas seccionadoras 189L-2 y 189L-5 para el interruptor 152L y, 189M-2 y 189M-3 para el interruptor $152 \mathrm{M}$ se consideran siempre cerradas.

Tal y como se puede observar en las Figuras 5 y 6 , el tiempo de duración es de 1,5 s. Además, la falla se producen después de $450 \mathrm{~ms}$ de haber iniciado
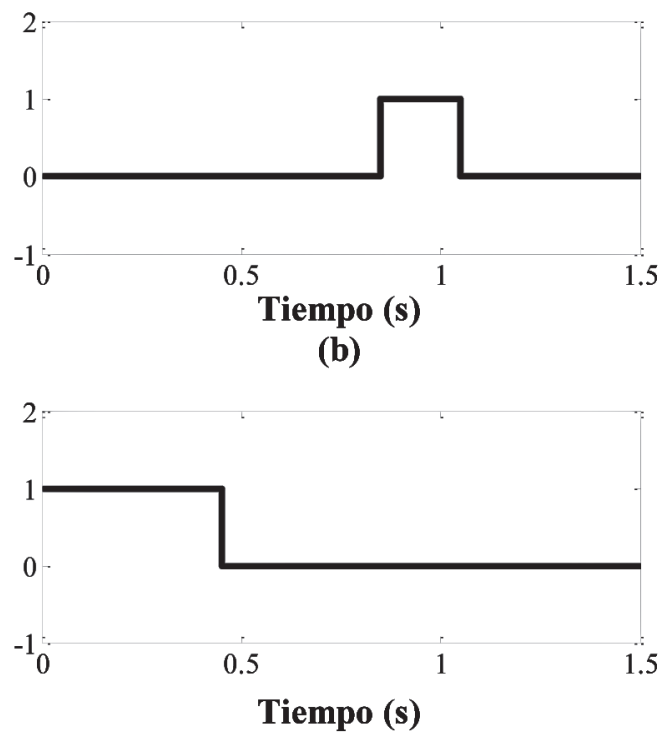

(d)

Figura 5. Señales lógicas cuando el interruptor 152L opera normalmente (a) falla simulada, (b) señal de auto-recierre, (c) posición del interruptor 152L, (d) posición del interruptor 152M. 


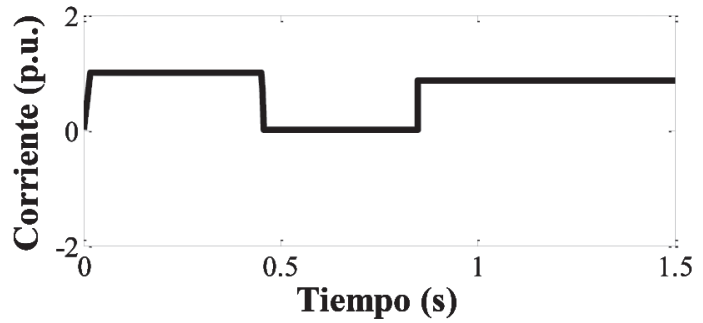

(a)

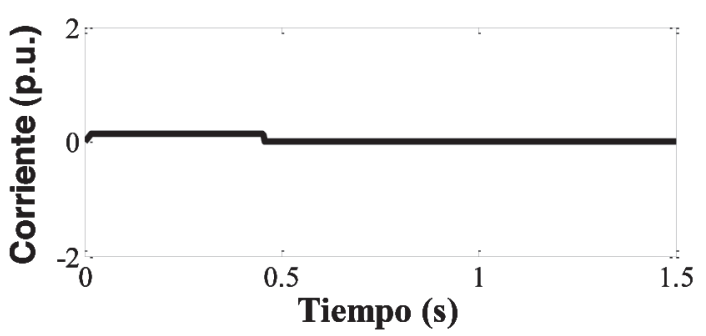

(c)

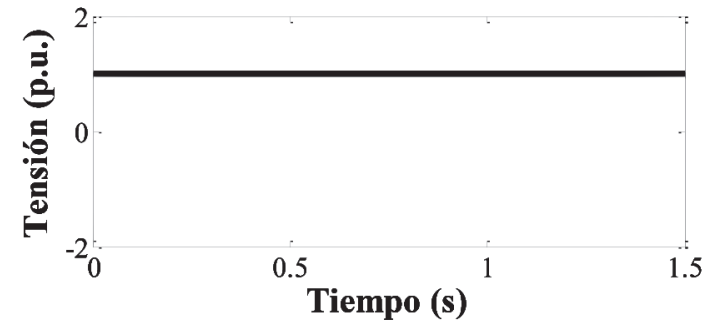

(b)

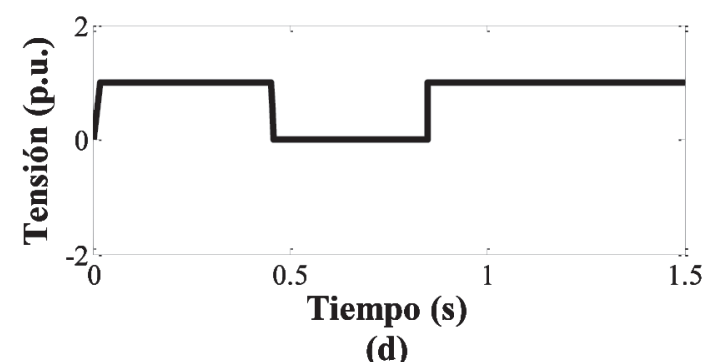

(d)

Figura 6. Flujos de corriente y tensiones eléctricas durante la operación normal (a) flujo de corriente a través de 152L, (b) tensión eléctrica en la Barra A, (c) flujo de corriente a través de 152M, (d) tensión eléctrica en el interruptor 152M.

Fuente: Elaboración propia, 2012.

las simulaciones, la señal de recierre automático se activa a $0,85 \mathrm{~s}(0,4 \mathrm{~s}$ después de la señal de disparo).

La Figura 5(a) representa la señal de disparo del dispositivo de protección, mientras que, Figura 5(b) representa la señal de auto-recierre enviada por el función de auto-recierre. De la Figura 5(c) es evidente que el interruptor 152L es abierto, debido a la señal de disparo, y luego se cierra debido a la señal de auto-recierre. En la Figura 5(d), se puede observar que el interruptor $152 \mathrm{M}$ es abierto debido a la señal de disparo y se mantiene abierto. Este interruptor deberá ser cerrado mediante un cierre manual (el cierre manual no se consideran en este artículo). De la Figura. 5, se puede concluir que la lógica implementada funciona correctamente cuando el sistema está funcionando sin ningún interruptor en mantenimiento.

Tal y como se puede notar en la Figura 6, la corriente fluye a través del interruptor $152 \mathrm{~L} \mathrm{y}$ $152 \mathrm{M}$ durante la operación sin falla. Después de la falla, ambos interruptores son abiertos, es decir, no hay corriente fluyendo a través de ninguno de los interruptores. Cuando la señal de auto-recierre es recibida, toda la corriente suplida a través de la "Línea 1" es brindada a través del interruptor $152 \mathrm{~L}$. El interruptor $152 \mathrm{M}$ se mantiene abierto y la corriente a través de éste es cero. Respecto a la tensión eléctrica, esta es constante en la "Barra A", pero no así en el interruptor 152M, debido a que este fue aislado.

\subsection{Simulaciones con el Interruptor de línea 152L en mantenimiento}

En este nuevo set de simulaciones, se considera que el interruptor 152L está abierto debido a labores de mantenimiento sobre el mismo. Consecuentemente, el personal de mantenimiento, ayudado por los operadores del SEN, abrirán ambas cuchillas seccionadoras 189L-2 y 189L-5. Esto se hace debido a medidas de seguridad implementadas en el SEN. Por lo tanto, las cuchillas seccionadoras 189L-2 y 189L5 para el interruptor 152L se consideran abiertas. Por otro lado, las cuchillas seccionadoras $189 \mathrm{M}-2$ y $189 \mathrm{M}-3$ para el interruptor $152 \mathrm{M}$ se consideran siempre cerradas. Basados en estas consideraciones, el algoritmo propuesto utilizará momentáneamente el interruptor $152 \mathrm{M}$, como 


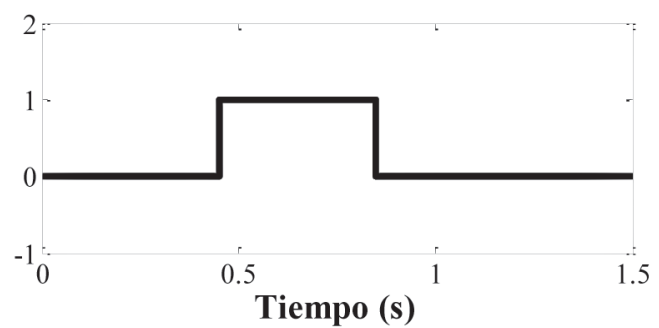

(a)

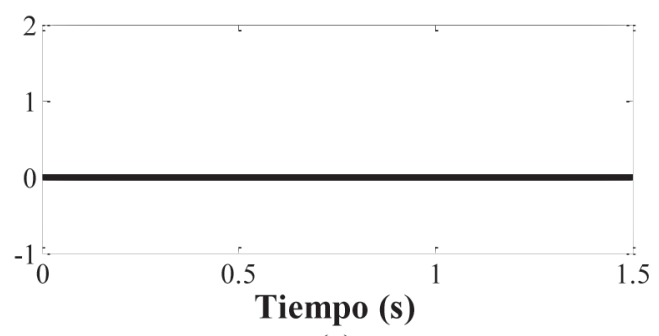

(c)

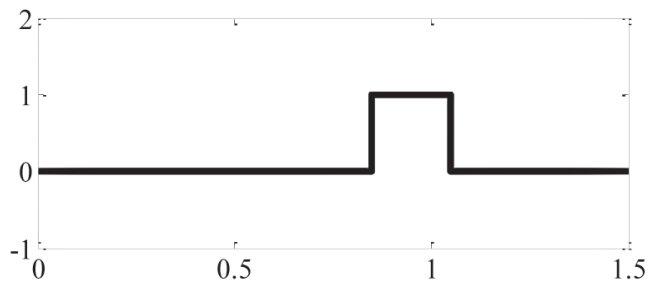

Tiempo (s)

(b)

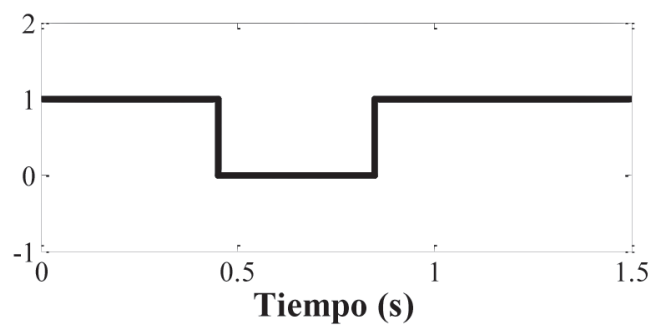

(d)

Figura 7. Señales lógicas cuando el interruptor 152L está en mantenimiento (a) falla simulada, (b) señal de auto-recierre, (c) posición del interruptor 152L, (d) posición del interruptor 152M.

Fuente: Elaboración propia, 2012.

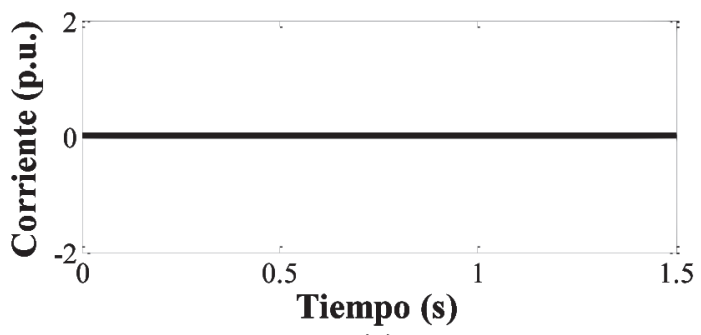

(a)

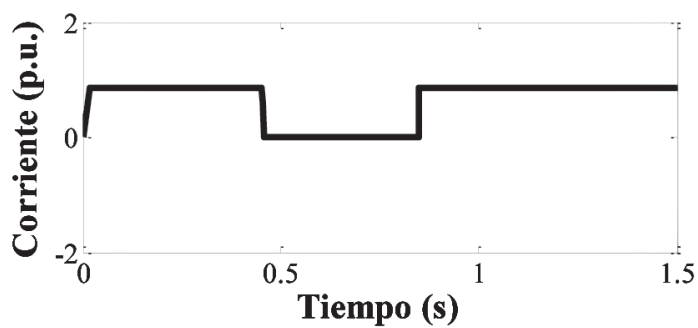

(c)

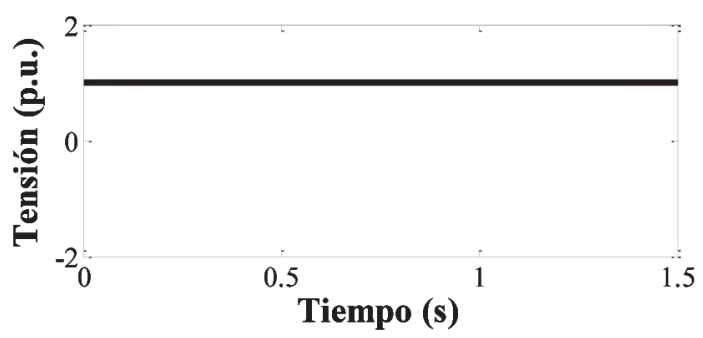

(b)

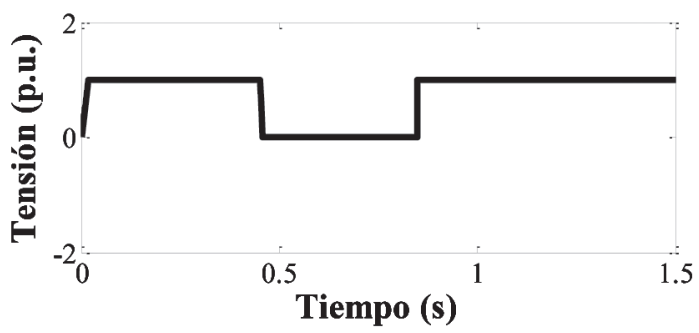

(d)

Figura 8. Señales lógicas cuando el interruptor 152L está en mantenimiento (a) flujo de corriente a través de 152L, (b) tensión eléctrica en la Barra A, (c) flujo de corriente a través de 152M, (d) tensión eléctrica en el interruptor 152M.

Fuente: Elaboración propia, 2012. 
un interruptor de línea, y así poder reconectar la "Línea 1".

De manera similar al caso presentado anteriormente en la Sección 5.1, la de duración de las simulaciones es de 1,5 s. Además, la falla se producen después de $450 \mathrm{~ms}$ de haber iniciado las simulaciones, la señal de recierre automático se activa a $0,85 \mathrm{~s}(0,4 \mathrm{~s}$ después de la señal de disparo).

La Figura 7(a) representa la señal de disparo del dispositivo de protección, mientras que, Figura 7(b) representa la señal de auto-recierre enviada por el función de auto-recierre. De la Figura 7(c) es evidente que el interruptor $152 \mathrm{~L}$ se encuentra abierto durante toda la simulación. En la Figura $7(\mathrm{~d})$, se puede observar que el interruptor $152 \mathrm{M}$ es abierto debido a la señal de disparo y es auto-recerrado cuando la señal de auto-recierre es recibida mediante del algoritmo propuesto. De la Figura. 7, se puede concluir que la lógica implementada funciona correctamente cuando el interruptor $152 \mathrm{~L}$ se encuentra fuera de operación.

Tal y como se puede notar en la Figura 8(a), la corriente a través del interruptor $152 \mathrm{~L}$ es nula durante las simulaciones. Esto confirma también que dicho interruptor está abierto. Por otro lado, es evidente en la Figura 8(c) que la corriente que fluye a través la "Línea 1 " es suplida a través del interruptor $152 \mathrm{M}$. Tal y como se espera, después de la falla, el interruptor $152 \mathrm{M}$ es abierto, es decir, no hay corriente fluyendo a través del interruptor. Cuando la señal de auto-recierre es recibida, toda la corriente suplida a través de la "Línea 1 " es brindada a través del interruptor $152 \mathrm{M}$. El interruptor $152 \mathrm{~L}$ se mantiene abierto y la corriente a través de este siguen siendo cero. Respecto a la tensión eléctrica, esta es constante en la "Barra A", pero no así en el interruptor $152 \mathrm{M}$, debido a que este fue aislado.

\section{CONCLUSIONES}

Una nueva y práctica metodología, para la función de auto-recierre en subestaciones de interruptor y medio del Sistema Eléctrico Nacional, fue presentada en este artículo de investigación. Se propuso controlar el interruptor del medio, o compartido, de manera tal que ejecute un ciclo de auto-recierre sobre este interruptor únicamente cuando un interruptor de línea se encuentra fuera de funcionamiento.

Basados en el monitoreo de la posición doble de las cuchillas seccionadoras del interruptor de línea y del interruptor del medio, dicho control fue realizado efectivamente. Se espera que en sistemas de potencia reales dicho método se siga implementando. En sistemas reales, el uso del protocolo de comunicación IEC 61850 para la automatización de subestaciones, y tomando en cuenta el uso de dispositivos electrónicos inteligentes, permite monitorear y controlar los interruptores.

Basado en las simulaciones obtenidas, las cuales intentan reproducir la operación del método en sistemas reales, así como el hecho que dicho algoritmo se encuentra funcionando en ciertas subestaciones del Sistema Eléctrico Nacional, una mejor maniobrabilidad de las subestaciones de interruptor y medio puede ser obtenida. Por lo tanto, ante posibles obras de mantenimiento sobre un interruptor de línea, el interruptor compartido realizará un auto-recierre y preservará la integridad del sistema de potencia, cuando ocurre una perturbación monofásica en la línea de transmisión.

\section{REFERENCIAS BIBLIOGRÁFICAS}

Phadke, A; Thord, J. (2009). Computer Relaying for Power Systems. Virginia: John Wiley \& Sons, Ltd.

Phadke, A; Horowitz, S. (2008). Power System Relaying. Virginia: John Wiley \& Sons, Ltd.

D. Nack. (2005). Reliability of substation configurations. Iowa: Iowa State University.

ICE-APMG. (2011). Estudio para revisión de filosofia de recierre en esquemas de interruptor y medio. San Jose: Grupo ICE

ICE-CENCE. (2012). ICE-CENCE. San Jose: Grupo ICE

IEC. (2004-05). Communication networks and systems in substations, IEC STD 61850.

Cordero Leiton, J. (2009). Protecciones del Sistema de Potencia. San Jose: Universidad de Costa Rica. 
Grigsby, L. (2006). Electric Power Engineering Handbook. Oxford:Taylor \& Francis Group. Mackiewicz, R. (2006, Oct. 29-Nov 1). Technical Overview and Benefits of the IEC 61850 Standard for Substation Automation. Paper presented at the Proceedings of the 2006 Power Systems Conference and Exposition, IEEE.

\section{NOMENCLATURA}

CENCE Centro Nacional de Control de Energía ICE Instituto Costarricense de Electricidad
IEC International Electrotechnical Commission p.u. Entiéndase por unidad

SEN Sistema Eléctrico Nacional

UEN Unidad Estratégica de Negocios

\section{SOBRE EL AUTOR}

Jairo Quirós-Tortós, Profesor Interino en el Departamento de Máquinas Eléctricas y Sistemas de Potencia, Escuela de Ingeniería Eléctrica, Universidad de Costa Rica. Estudiante de doctorado en la Universidad de Manchester, Inglaterra.

Correo electrónico: jairoquirostortos@ieee.org 
